Supplementary Materials

pertaining to

High economic inequality makes us feel less wealthy

Note. In this file, we present additional details about participants in studies 1-6 (section S1) perceived poverty of the group in studies $3,4,5$ and 6 (section S2), as well as additional details about mediation analyses in Experiment 7 (section S3). 


\section{Section 1}

Participants in Experiment 1. Ninety-seven undergraduate students of a Spanish university took part in this experiment in exchange for course credit. Data were collected at one point in time. Three participants were excluded from the data analyses because their native language was not Spanish. The final sample consisted of 94 participants (72 women) aged between 19 and 47 years $(M=21.55, S D=3.89)$. Forty-eight participants were randomly assigned to the high economic inequality condition, and 46 participants were assigned to the low economic inequality condition. A sensitivity analysis for a two-tailed independent samples t-test with this sample size set at an alpha at .05 showed that this experiment was powerful enough to find an effect size of $d=.58$, with .80 statistical power. Data from this experiment were also used in Experiment 2, reported in the paper by Citation Blinded to test another hypothesis.

Participants in Experiment 2. Two hundred and eleven undergraduate students of a Spanish university took part in this experiment in exchange for course credit. Data were collected at two points because data collection could not be completed before the end of the semester. Five participants were excluded from the data analyses because they were not native Spanish speakers. The final sample consisted of 206 participants (170 women) aged between 18 and 37 years $(M$ $=19.99, S D=2.81)$. One hundred and four participants were assigned to the high economic inequality condition, and 102 participants were assigned to the low economic inequality condition. A sensitivity analysis for a two-tailed independent samples t-test with this sample size set at an alpha at .05 showed that this experiment was powerful enough to find an effect size of $d=.39$, with .80 statistical power. Data from this experiment were also used in Experiment 1, reported in the paper by Citation Blinded, to test another hypothesis.

Participants of Experiment 3. Sixty undergraduate students (37 women, 22 male, and 1 unreported) of an Australian university took part in this experiment in exchange for course credit. Data were collected at one point in time. Their ages ranged from 16 to 23 years $(M=18.45, S D$ $=1.25$ ). Thirty-four participants were assigned to the high economic inequality condition and 26 participants were assigned to the low economic inequality condition. A sensitivity analysis for a two-tailed independent samples t-test with this sample size set at an alpha at .05 showed that this 
experiment was powerful enough to find an effect size of $d=.74$, with .80 statistical power. Data from this experiment were also used in Experiment 2, reported in the paper by Citation Blinded, to test another hypothesis.

Participants in Experiment 4. Two hundred and nine individuals from the United States participated in the experiment via Amazon's Mechanical Turk (Buhrmester, Kwang, \& Gosling, 2011) in exchange for $1 \$$. Data were collected at one point in time. Eleven participants were excluded from the data analysis: six of them because they did not finish the experiment, three because they were not native English speakers, and two because they failed to report the income group they were assigned to. The final sample consisted of 198 participants ( 88 women, 109 men, and 1 unknown) ranging from 18 to 67 years $(M=33.6, S D=10.10)$. One hundred and one participants were assigned to the high economic inequality condition and 97 were assigned to the low economic inequality condition. A sensitivity analysis for a two-tailed independent samples ttest with this sample size set at an alpha at .05 showed that this experiment was powerful enough to find an effect size of $d=.40$, with .80 statistical power. Data from this experiment were also used in Experiment 3, reported in the paper by Citation Blinded, to test another hypothesis.

Participants in Experiment 5. One hundred and one undergraduate students of a Spanish university accepted to participate in this experiment in exchange for course credit. Data were collected at one point in time. Three participants were excluded because their native language was not Spanish. The final sample included 98 participants ( 82 women) aged between 18 and 29 years $(M=20.20 ; S D=1.42)$. Fifty participants were assigned to the high economic inequality condition and 48 participants were assigned to the low economic inequality condition. A sensitivity analysis for a two-tailed independent samples t-test with this sample size set at an alpha at .05 showed that this experiment was powerful enough to find an effect size of $d=.57$, with .80 statistical power. Data from this experiment were also used in Experiment 1, reported in the paper by Citation Blinded to test another hypothesis.

Participants in Experiment 6. Ninety-five undergraduate students of a Spanish university participated in this Experiment in exchange for course credit. Data were collected at one point in time. Four participants were excluded because their native language was not Spanish. The final 
sample included 91 participants ( 78 women) aged between 18 and 42 years $(M=20.38 ; S D=3.39)$. Forty-six participants were assigned to the high economic inequality condition and 45 participants were assigned to the low economic inequality condition. A sensitivity analysis for a two-tailed independent samples t-test with this sample size set at an alpha at .05, showed that this experiment was powerful enough to find an effect size of $d=.59$ with .80 statistical power. Data from this experiment were also used in Experiment 2, reported in the paper by Citation Blinded, to test another hypothesis. 


\section{Section 2}

Study 3 and 4

We measured poor perceptions of the own group: (i.e., "How poor is your group?”)

Participants responded to each item on a scale ranging from 1 (very poor) to 9 (very wealthy).

Results of study 3 showed no differences between conditions in the group's poverty: $F(1,58)=$ $2.13, p=.149, \eta^{2}=.036$.

Results of study 4 showed no differences between conditions in the group's poverty: $F(1,196)=$ $.20, p=.650, \eta^{2}=.001$.

Study 5 and 6

We measured poor perceptions of the own group (i.e. "How poor is your group?" $(1=$ not at all, 9 = very much).

Results of study 5 showed no differences between conditions in the group's poverty: $F(1,96)=$ $1.19, p=.277, \eta^{2}=.012$

Results of study 6 showed no differences between conditions in the group's poverty $F(1,89)=$ $1.96, p=.165, \eta^{2}=.022$ 


\section{Section S3}

Mediation analysis. We performed these analysis using the Process macro for SPSS (Model 4), bootstrapping for 10,000 resamples and with a 95\% confidence interval (Hayes, 2013).

We conducted three mediation analyses to check if the effect of economic inequality (coded as $0=$ Low, $1=$ High) on perceived wealth was mediated by (1) relative deprivation, (2) upward and downward comparison, and/or (3) upward and downward similarity. Results showed that the indirect effects of relative deprivation (indirect effect $=-0.03(0.03), 95 \%$ $\mathrm{CI}=[-.1074, .0068])$, upward comparison $($ indirect effect $=-0.05(0.04), 95 \% \mathrm{CI}=$ $[-.1377, .0144])$, downward comparison $($ indirect effect $=0.02(0.02), 95 \% \mathrm{CI}=[-.0181$, $.0885])$, and upward similarity (indirect effect $=-0.02(0.03), 95 \% \mathrm{CI}=[-.1067, .0482])$ were not different from zero. Only the indirect effect of downward similarity was different from zero (indirect effect $=0.05(0.03), 95 \% \mathrm{CI}=[.0092, .1349])$. However, it should be noted that the indirect effect was in the opposite direction than the direct effect (direct effect $=-0.33(0.12), 95 \% \mathrm{CI}=[-.5592,-.1012])$.

Even though we did not find an effect of economic inequality effect on perceived poverty, we conducted the mediation analyses as planned given that indirect and direct effects can be significant even when the total effect is not (Rucker, Preacher, Tormala, \& Petty, 2011). Therefore, we conducted three mediation analyses using (1) relative deprivation, (2) upward and downward comparison, and (3) upward and downward similarity as mediator variables.

Results showed that the indirect effect via relative deprivation was significantly different from zero (indirect effect $=0.11(0.06), 95 \% \mathrm{CI}=[.0241, .2645])$, suggesting that high (vs. low) economic inequality leads to perceived poverty in the ingroup through the mediation of relative deprivation. However, the direct effect, similarly to the total effect, was not significant, direct effect $=0.13(0.21), 95 \% \mathrm{CI}=[-.2913, .5505])$. The indirect effects via upward comparison (indirect effect $=-0.07(0.07), 95 \% \mathrm{CI}=[-.2148, .0522])$ and downward 
comparison (indirect effect $=-0.06(0.06), 95 \% \mathrm{CI}=[-.1973, .0554])$ were not different from zero. Regarding similarity, results showed that upward similarity was not different from zero (indirect effect $=-0.08(0.08), 95 \% \mathrm{CI}=[-.2444, .0694])$ but the indirect effect via downward similarity was significantly different from zero (indirect effect $=-0.13(0.06), 95 \%$ $C I=[-2916,-.0364])$. The direct effect of this mediation model was positively significant (direct effect $=0.45(0.23), 95 \% \mathrm{CI}=[.0080, .8967])$. Therefore, when we considered downward similarity, high (vs. low) economic inequality led participants to perceive their ingroup as poorer via a direct effect. However, suggesting suppression, the indirect effect was in the opposite direction (Rucker et al., 2011). That is, high (vs. low) economic inequality led participants to perceive their group as less similar to the poorest group, which in turn reduced perceived ingroup poverty. Even though these mediational effects are interesting, we treat them caution because there were only a limited number of mediational effects and we cannot rule out that these represent Type 1 error. 


\section{References}

Buhrmester, M., Kwang, T., \& Gosling, S. D. (2011). Amazon's Mechanical Turk: A new source of inexpensive, yet high-quality, data? Perspectives on Psychological Science, 6, 3-5. doi: $\underline{10.1177 / 1745691610393980}$

Hayes, A. F. (2013). An introduction to mediation, mode ration, and conditional process analysis. New York: The Guilford

Rucker, D. D., Preacher, K. J., Tormala, Z. L., \& Petty, R. E. (2011). Mediation Analysis in Social Psychology: Current Practices and New Recommendations, Social and Personality Psychology Compass 6, 359-371. https://doi.org/10.1111/j.17519004.2011.00355.x 